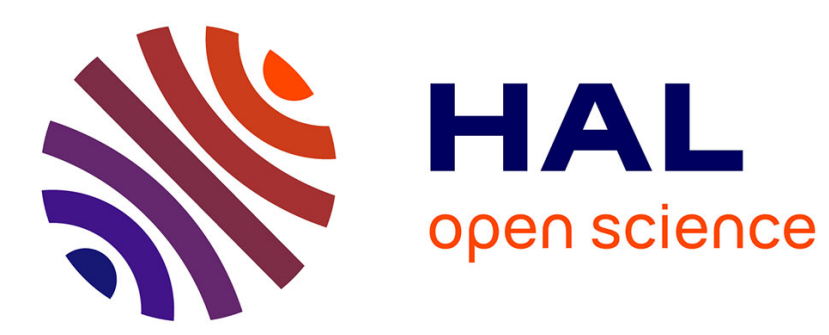

\title{
Basileis e euergesia in Aristotele: Politica V 10, 1310b 34-40 e l'exemplum di Codro \\ Cesare Zizza
}

\section{To cite this version:}

Cesare Zizza. Basileis e euergesia in Aristotele: Politica V 10, 1310b 34-40 e l'exemplum di Codro. Archimède: archéologie et histoire ancienne, 2014, 1, pp.114-123. halshs-01586163

\section{HAL Id: halshs-01586163 \\ https://shs.hal.science/halshs-01586163}

Submitted on 12 Sep 2017

HAL is a multi-disciplinary open access archive for the deposit and dissemination of scientific research documents, whether they are published or not. The documents may come from teaching and research institutions in France or abroad, or from public or private research centers.
L'archive ouverte pluridisciplinaire HAL, est destinée au dépôt et à la diffusion de documents scientifiques de niveau recherche, publiés ou non, émanant des établissements d'enseignement et de recherche français ou étrangers, des laboratoires publics ou privés. 


\section{VARIA}

coord. Maria Teresa Schettino

\section{BASILEIS E EUERGESIA IN ARISTOTELE: POLITICA V 10, 1310B 34-40 E L'EXEMPLUM DI CODRO}

\section{Cesare ZIZZA}

Cesare Zizza

RÉSUMÉ

Le but de mon travail est d'analyser les considérations formulées par Aristote sur le rapport entre basileia et aristocratia et entre basileia et euergesia (Politique V 10, 1310b 2-3, 9-12, 31-37). J'essaierai, en outre, de reconstruire l'image qu'Aristote nous donne de Codros (V 10, 1310b 36-37) et, à travers I'analyse de l'exemplum relatif au roi mythique d'Athènes, je tenterai de rectifier l'interprétation que I'on donne en général des lignes $1310 \mathrm{~b}$ 34-36 et j'essaierai de rendre compte des relations complexes qu'Aristote établit entre «faits» (exempla) et concepts.
MotS-CLÉS

Aristote,

politique

monarchie,

tyrannie,

basileia/royauté,

euergesia,

exemplum,

Codros.
Scopo del mio lavoro è analizzare le considerazioni formulate da Aristotele sul rapporto tra basileia e aristocratia e tra basileia e euergesia (Politica V 10, 1310b 2-3, 9-12, 31-37). Proverò, inoltre, a ricostruire I'immagine che Aristotele ci fornisce di Codro (V 10, 1310b 36-37) e, attraverso I'analisi dell'exemplum relativo al mitico re di Atene, tenterò di rettificare l'interpretazione che in genere viene data alle linee 1310b 34-36, mettendo in evidenza il complesso sistema di relazioni che Aristotele stabilisce tra "fatti" (exempla) e 'concetti'.
The aim of my paper is to examine the relation between basileia and aristokratia and between basileia and euergesia (Politics V 10, 1310b 2-3, 9-12, 31-37). Furthermore, I will try to reconstruct Aristotle' depiction of Codrus (V 10, 1310b 36-37) and through an analysis of the exemplum concerning the mythical king of Athens, to revise the current interpretation of $\mathrm{V} 10$, $1310 \mathrm{~b} 34-36$ and to give an account of the complex relationship established by Aristotle between "facts" (exempla) and 'ideas'. 
Il brano che analizzeremo nel presente lavoro ( $V$ 10, 1310b 34-40) - all'interno del quale compare, tra i rebenefattori, I'exemplum di Codro - corrisponde ad uno dei primi segmenti argomentativi del discorso peri monarchias che Aristotele affronta a partire dal capitolo 10 del V libro della Politica [1]: prosegue, qui, I'indagine

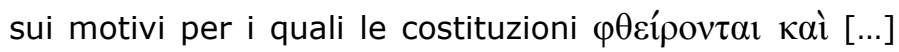

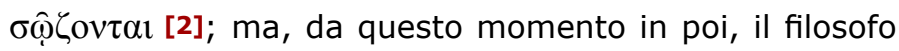
concentra la sua attenzione sui regimi di tipo monarchico, lasciando da parte le altre politeiai, delle quali si era occupato nei capitoli precedenti.

Nonostante le indicazioni programmatiche poste in apertura del capitolo facciano un esplicito riferimento al tema della rovina e della salvezza della basileia e della tyrannis (1310a 39-40), la questione specifica è trattata solo al termine di una sezione - per così dire - introduttiva (1310a 40-1311a 25), nella quale Aristotele sembra voler richiamare alla mente del suo pubblico quanto detto nel corso dell'opera sulle dinamiche per le quali le

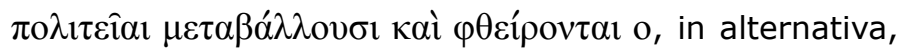

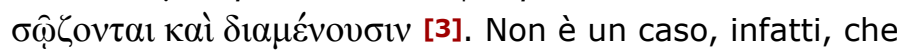
all'inizio e alla fine di questa 'premessa' venga ribadito che, per quanto riguarda le archai $\tau \hat{\omega} v \mu \varepsilon \tau \alpha \beta \delta \lambda \hat{\omega} v$, ciò che si può dire sui regimi monarchici è approssimativamente analogo a quanto rilevato a proposito di tutte le altre co-

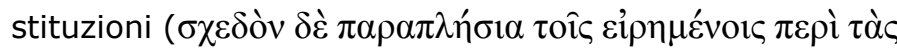

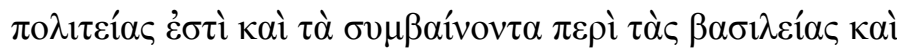

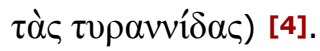

\section{BASILEIA E TYRANNIS A CONFRONTO}

A questo punto, avendo introdotto l'argomento in maniera tale da rendere coerente e coeso il ragionamento fin qui seguito - e di qui in poi portato avanti fino alla

[1] L'edizione critica utilizzata è quella di Ross 1957. Per il commento ai brani del $\mathrm{V}$ libro, che qui vengono solo segnalati, si rimanda al volume di prossima pubblicazione presso i tipi de «L'Erma» di Bretschneider (Roma), a cura di M. Curnis, M. E. De LunA, C. Zizza: Aristotele. La Politica. Libri V-VI (dir. L. Bertelli \& M. Moggi).

[2] Arist., Pol. V 9, 1310a 36-38. Di qui in poi, i riferimenti non corredati da alcuna indicazione aggiuntiva si intendano come rinvii al testo della Politica; quelli accompagnati solo dal titolo come rimandi ad opere del corpus aristotelico. A meno che motivi di chiarezza non abbiano indotto a fare diversamente, di norma, i loci del capitolo 10 del V libro della Politica sono stati citati - soprattutto nel testo, più raramente nelle note - senza l'indicazione del libro e del capitolo.

[3] Le citazioni sono tratte dal brano segnalato alla nota precedente.

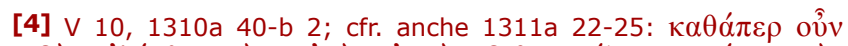

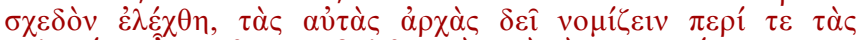

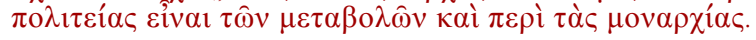

[5] V 10, 1310b 2-7 (la traduzione è mia; lo stesso dicasi - in assenza di indicazioni contrarie - per i brani della Politica citati nel corso del lavoro). fine del libro -, Aristotele passa a menzionare, nell'ordine, I'aristokratia, I'oligarchia e la demokratia; quindi, presenta una serie di associazioni tra queste politeiai e le due monarchiai: «il regno è conforme all'aristocrazia, mentre la tirannide è una combinazione della oligarchia

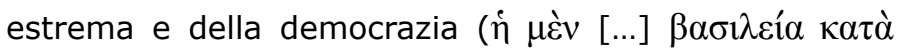

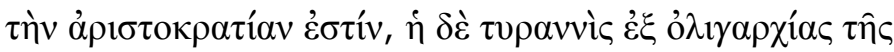

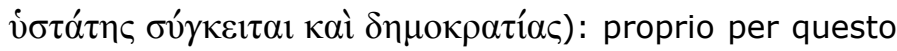
la tirannide è anche il regime politico più dannoso per

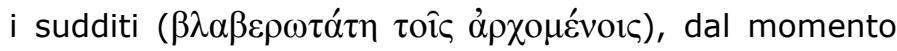

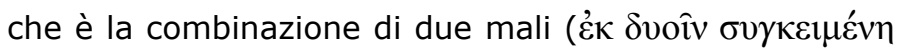
$\kappa \alpha \kappa \omega \hat{v})$ e ha in sé le deviazioni e gli errori derivanti da

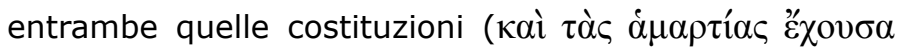
$\tau \grave{\alpha} \varsigma \pi \alpha \rho ’ \grave{\alpha} \mu \varphi 0 \tau \dot{\varepsilon} \rho \omega v \tau \hat{\omega} v \pi 0 \lambda \imath \tau \varepsilon \imath \hat{\omega} v) \gg$ [5].

Diversamente dalla seconda associazione (tyrannis-oligarchia-demokratia), la prima (basileia-aristokratia) non risulta corredata da alcun tipo di giudizio. Nelle linee che seguono, tuttavia, non mancano suggerimenti sulla valutazione che il filosofo dava a entrambe le monarchiai e che nel prosieguo dell'analisi verrà espressa sempre più a chiare lettere: la basileia costituisce il tipo di monarchia più 'salutare' per i sudditi non solo perché opposta e contraria alla tirannide (1310b 8) - che è appunto detta essere più dannosa (1310b 5) -, ma anche perché se la basileia è posta sullo stesso piano dell'aristocrazia (ed è, pertanto, valutata alla stregua della politeia che per definizione rappresenta il governo migliore perché gestito dai migliori e in vista del meglio) [6], la tirannide, dal canto suo, oltre ad avere i mali della oligarchia estrema e della democrazia, è messa in relazione con due politeiai che già di per sé non erano certo considerate dallo Stagirita come sinonimi di buon governo [7].

Così strutturato, questo primo confronto tra regno e tirannide si rivela funzionale a fornire le basi alle informazioni che seguono e che - nello specifico - continuano

[6] Sulle assonanze esistenti tra le due politeiai (basileia e aristokratia), che, altrove, sono definite orthai (in entrambi i casi, infatti, il potere è in mano a individui virtuosi, a prescindere dal numero di questi), cfr. soprattutto III 7, 1279a 32-40; 18, 1288a 32-b 2; IV 2, 1289a 30-35. Su quanto detto fin qui si vedano AuBonNET 1989, p. 204; SchütrumpF \& Gehrke 1996, p. 545-547, 550; KeYt 1999, p. 147-148; ZizZa 2012a, p. 170-171; ACCATtino \& CURnis 2013, p. $179-182,240-243$.

[7] Cfr. V 10, 1311a 8-20. È qui che I'argomento riceve una trattazione più adeguata e le parekbaseis e le hamartiai derivanti alla tirannide vengono chiamate per nome e, talvolta, corredate da cursori - ma efficaci - riferimenti a specifiche strategie adottate in tutte e tre i regimi menzionati. Nella fattispecie, si tratta di indicazioni di natura esemplificativa che si rivelano funzionali alla dimostrazione della tesi enunciata alle linee 1310b 5-6 (cfr. supra, nel testo): la tirannide è la peggiore tra tutte le costituzioni deviate perché combina insieme i mali di almeno altri due regimi appartenenti ad una medesima categoria di politeiai, quelle - cioè - degenerate (le cosiddette 'tirannidi condivise'). Al riguardo, cfr., per esempio, IV 2, 1289a 35-b 3; 4, 1292a 17-18; V 10, 1312a 40-b7 (con ZizZA 2012a, p. 163-165); 1312b 34-38; 11, 1313b 32-33; VI 4, 1319b 27-28. 
la serie di correlativi ( $\dot{\eta} \mu \grave{\varepsilon} v \beta \alpha \sigma \imath \lambda \varepsilon^{\prime i} \alpha$ / $\dot{\eta} \delta \grave{\varepsilon} \tau v \rho \alpha v v \grave{c} \varsigma$ ) sul tema della $\gamma \varepsilon ́ v \varepsilon \sigma i \varsigma$, delle finalità e delle caratteristiche delle due monarchiai: «Ciascuno dei due tipi di monarchia trae direttamente origine da motivazioni op-

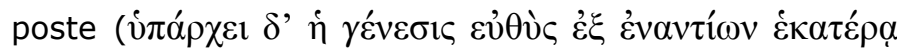
$\tau \hat{\omega} v \mu$ ov $\alpha \rho \chi(\hat{\omega} v)$ : il regno, infatti, è sorto per la difesa

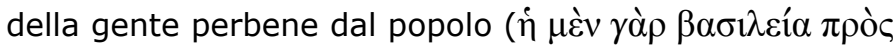

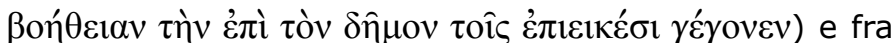

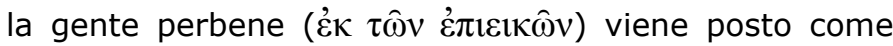
re colui che è superiore per virtù o per azioni derivanti

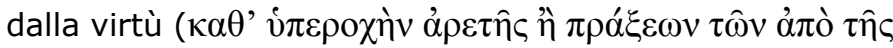

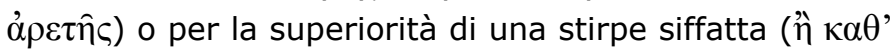

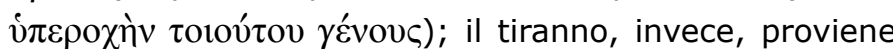
dal popolo e dalla massa contro i notabili (ó $\delta \varepsilon \grave{\varepsilon} \tau u ́ p \alpha v v o \varsigma$

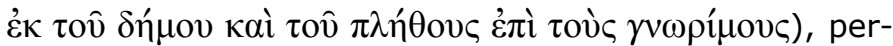
ché il popolo non subisca alcun torto da costoro (ö $\pi \omega \varsigma$ o

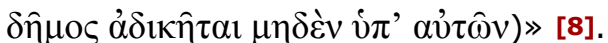

Gli gnorimoi (cioè i notabili) e, in generale, i sudditi che per qualche motivo eccellono sono invisi alla tirannide [9]; la basileia, invece, è stata istituita per comune volere degli epieikeis (cioè la gente perbene, i migliori, gli uomini eccellenti) [10] ed è stata sempre gestita da individui appartenenti a questa stessa categoria di aristoi per

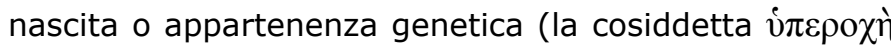

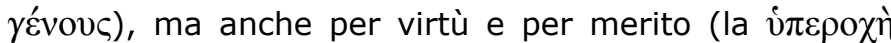

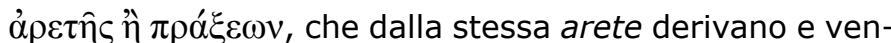
gono ispirate e guidate). Identica contrapposizione per quanto riguarda le finalità perseguite dall'uno e dall'altro monarchos e, in particolare, per quanto riguarda il rapporto che ciascuno tende a stabilire con il demos: se la basileia trova la sua ragion d'essere nella difesa degli interessi degli epieikeis nei confronti del demos (e si pone, dunque, come obiettivo la promozione e la valorizzazione dell'arete che il popolo in quanto plethos - massa indistinta - non possiede), la tyrannis, invece, è proprio sul demos/plethos che fa leva per conquistare il governo di una polis e per imporsi non solo sugli stessi elementi dai quali ha origine (vale a dire, il demos e il plethos), ma anche sugli gnorimoi, demagogicamente e artatamente presentati come i maggiori responsabili delle ingiustizie subite dal popolo [11].

[8] V 10, 1310b 7-14

[9] Cfr. V 10, 1310b 14-16 (per il testo e la traduzione: infra, n. 19), ma anche V 10, 1311a 15-20. A questo proposito, si veda ZizzA 2012b, p. $179-185$.

[10] Questi epieikeis poco più avanti vengono definiti gnorimoi (cfr. 1310b 13). A questo proposito: KEYT 1999, p. 145.

[11] Cfr. anche V 10, 1310b 15-16 (riportato infra, n. 19).

[12] Nella Politica, il termine epieikes funziona come uno «specifico indicatore di ceto» (GASTALDI 1978, p. 89).

[13] Parte del testo (con traduzione) è riportato infra, n. 19.

[14] Nella fattispecie, il riferimento concerne diversi tipi di tyrannoi classificati in base alla loro 'provenienza': demagogos, basileus, demiourgos, theoros e, nelle oligarchie, colui che era chiamato a rivestire da solo archai
Evidentemente, I'accenno agli epieikeis, come gruppo dal quale proveniva il basileus [12], genera un discorso sul tyrannos strutturato in maniera simmetrica e speculare rispetto al primo. A sua volta, il riferimento alla coppia tyrannis-demos/plethos introduce una variazione all'alternanza fin qui adottata nel ragionamento $(\dot{\eta} \mu \dot{\varepsilon} v$

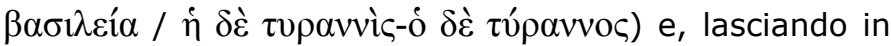
sospeso il discorso appena iniziato sulla basileia e sulle qualità possedute dal basileus, dà I'avvio a una digressione (1310b 14-31) [13], che si chiude con una breve rassegna di casi storici (1310b 26-31) e che risulta tutta dedicata ai tropoi attraverso i quali un tyrannos poteva diventare tale sfruttando un ruolo o un incarico ottenuti in maniera lecita e legittima [14].

\section{BASILEIS E EUERGESIA}

Dai tiranni - e, in particolare, da quelli citati come exempla (Fidone, Falaride, Panezio, Cipselo, Pisistrato, Dionisio) - si passa di nuovo ai basileis (1310b 31):

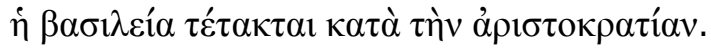

La ripetizione quasi alla lettera dell'enunciato posto in apertura del capitolo (cfr. 1310b 2-3: $\dot{\eta} \mu \grave{\varepsilon} v$ [...] $\beta \alpha \sigma \lambda \lambda \varepsilon i ́ \alpha$

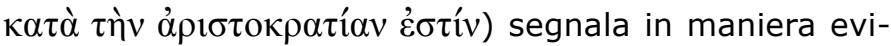
dente la ripresa di un argomento precedentemente solo accennato e l'inizio di una sezione interamente dedicata

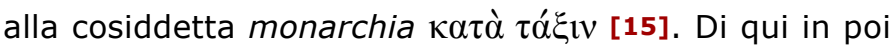
- tra riprese, aggiunte ed espressioni sinonimiche [16] , il senso e il valore della (ri)proposta associazione tra basileia e aristokratia risultano più chiari ed espliciti; il discorso sui basileis-epieikeis viene 'rimbastito', I'arete ulteriormente declinata [17] e la theoria coniugata con le praxeis: come nella rubrica precedente, anche in questa, compaiono degli exempla, questa volta costituiti da aristoi-basileis; si tratta, nella fattispecie, di Kodros (del quale ci occuperemo tra poco), di Kyros e dei non meglio identificati re dei Lacedemoni, dei Macedoni e dei Molossi.

Il locus di cui stiamo parlando e sul quale soffermeremo la nostra attenzione è il seguente (1310b 31-40):

importanti (al riguardo cfr. AuBOnNET 1989, p. 201-204; SCHÜTRUMPF \& GeHRKE 1996, p. 547-550; KEYT 1999, p. 145-146; ZizZA 2012b, p. 178179 e infra, n. 19).

[15] Cfr. Rhet. I 8, 1366a 1-2.

[16] Alle nozioni di arete e genos - che ricorrono sia a 1310b 11-12, sia a $1310 \mathrm{~b} 33$ - se ne aggiungono altre (axia, euergesia, dynamis: 1310b 33-34) non menzionate esplicitamente nella parte iniziale del capitolo 10 e, cioè, nel primo (breve) intervento sul tema della basileia (1310b 9-

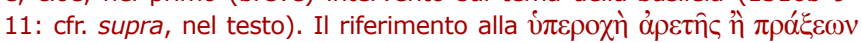
di 1310b 11 viene adesso reso più perspicuo da tutta una serie di casi concreti, giudicabili (e, di fatto, giudicati) come azioni 'buone', virtuose e vantaggiose per tutta la comunità (cfr., per il secondo intervento sul tema della basileia, 1310b 31-40: infra, nel testo).

[17] Al riguardo, si veda la nota precedente. 
«Come dunque abbiamo detto, il regno si colloca al li-

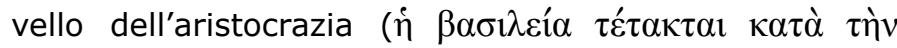

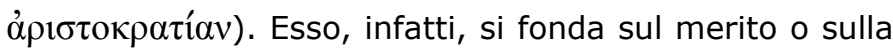
virtù personale o su quella della stirpe o sui benefici o su

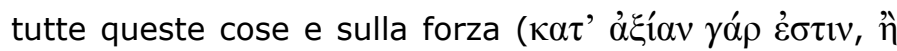

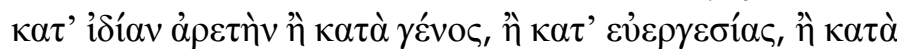

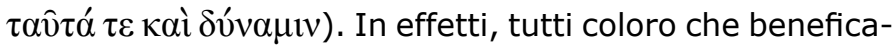

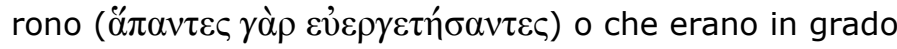
di benificare le città o i popoli ottenevano questo onore

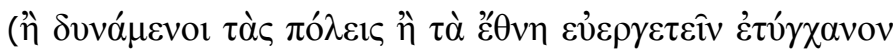
$\tau \hat{\imath} \varsigma \tau \iota \mu \hat{\eta} \varsigma \tau \alpha u ́ \tau \eta \varsigma)$ : alcuni, come Codro, per aver impedito che diventassero schiavi di guerra (oi $\mu \grave{\varepsilon} v \kappa \alpha \tau \grave{\alpha} \pi$ ó $\lambda \varepsilon \mu o v$

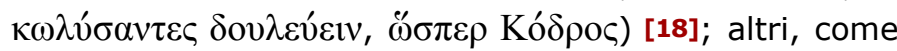
Ciro, per averli liberati (oi $\delta$ ' $̇ \lambda \varepsilon v \theta \varepsilon \rho \omega \sigma \sigma \alpha \nu \tau \varepsilon \varsigma$, Kôpoc), o - come i re dei Lacedemoni, dei Macedoni e dei Molossi - per aver fondato città o essersi imposses-

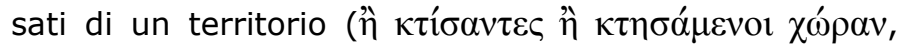

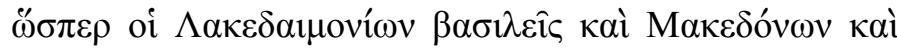

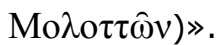

Diversamente dalle linee dedicate alla tirannide, a proposito della quale Aristotele opera una distinzione tra quelle più antiche (nate per 'degenerazione') e quelle più

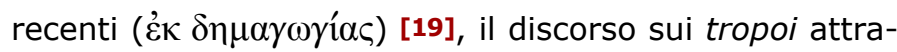
verso i quali un individuo perbene poteva diventare basileus risulta coniugato tutto al passato. Evidentemente, per la basileia ek ton epieikon (1310b 10) - considerata dallo Stagirita auspicabile, ma non realizzabile [20] - era possibile individuare degli esempi pertinenti solo andando molto indietro nel tempo: è così, infatti, che il filosofo riesce a individuare la maggior parte dei re menzionati a 1310b 37 s. (sono le ultime linee del brano citato sopra) e presentati come modelli di virtù difficilmente uguagliabili. D'altra parte, non è certamente un caso che la sezione storico-esemplificativa dedicata alla cosiddetta basileia 'aristocratica' presenti delle consonanze proprio con la monarchia basilike descritta nel III libro e collo-

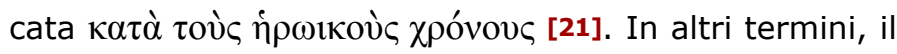
riferimento ai re-benefattori (Codro, Ciro, etc.) consente ad Aristotele di approfondire alcuni aspetti che - altrove, nella Politica - vengono riconosciuti come tratti tipici dei basileis di un passato ormai irrimediabilmente 'perdu-

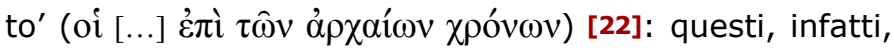
come del resto i re del brano in esame, godevano del consenso del popolo, perché, oltre a comportarsi secondo la legge (kata nomon), «erano stati benefattori della

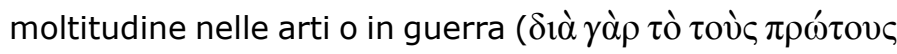

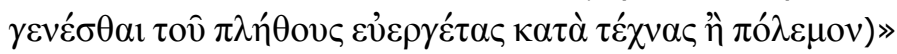
e «avevano», altresí, «unificato o esteso il territorio ( $\delta$ ì̀

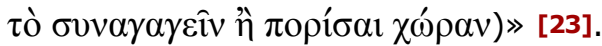

\section{BASILEIS E EUERGESIA: STRUTTURA E ARTICOLAZIONE DEL DISCORSO}

Come è evidente, nella rubrica - per così dire - peri monarchias aristokratikes gli esempi addotti sono in tut-

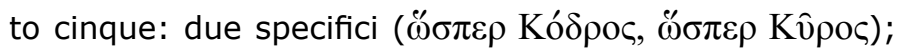
gli altri 'anonimi' e presentati in serie, uno di seguito all'altro (

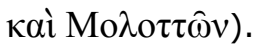

Eccezion fatta per questi ultimi exempla appartenenti a una medesima categoria di euergetesantes, per il resto, i casi storici (o mitistorici: Codro) menzionati non vengono a costituire un elenco compatto e coeso di nomi. Tra un exemplum e l'altro, infatti, compaiono alcuni segmenti concettuali che contribuiscono ad arricchire il

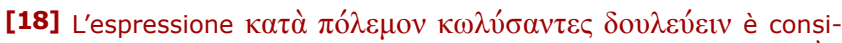
derata da NEWMAN 1902 [1973], p. 419 una variante della formula $\tau \grave{v} v$

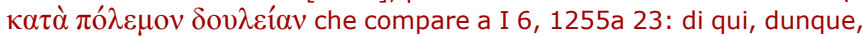
la traduzione da me proposta. Sul locus citato da Newman cfr. la traduzione e il commento di Besso \& Curnis 2011, p. 159 e 261-262. Per altre interpretazioni - più o meno condivisibili - cfr., per esempio, VIANO 1952 [2002], p. 463: «gli uni per aver impedito che la loro città fosse asservita ai nemici in seguito alla guerra, come Codro»; LAURENTI 1973 [2005], p. 182: «taluni li preservarono dalla schiavitù di guerra, come Codro»; García Gual \& PÉREZ JimÉnez 1986 [2003], p. 227 «unos con la guerra, librándolos de la esclavitud, como Codro»; AuBONNET 1989, p. 74: «soit qu'ils les aient préservés, après une guerre, de l'asservissement comme Codros»; SchütrumpF \& GeHRKE 1996, p. 75: «So bewahrten einige in Kriegen (ihr Volk) davor, (dem Gegner) als Sklave zu dienen, wie Kodros»; KEYT 1999, p. 24: «some, like Codrus, by preventing their enslavement in war»; LÓPEZ BARJA DE Quiroga \& García Fernández 2005, p. 308: «unos por haber impedido que fueran esclavizados en la guerra como Codro». Sulla questione specifica e per una discussione di queste e altre ipotesi interpretative si rinvia al commento al $\mathrm{V}$ libro della Politica di prossima pubblicazione (al riguardo: supra, n. 1).

[19] Il riferimento specifico è a 1310b 14-26: «infatti la maggior parte dei tiranni, più o meno, è uscita, per così dire, dalle file dei

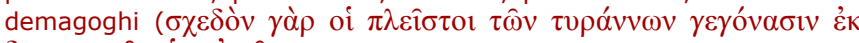
$\delta \eta \mu \alpha \gamma \omega \gamma \hat{\omega} \nu \hat{\omega} \varsigma \varepsilon i \pi \varepsilon \hat{\imath} v)$, i quali acquisirono credito grazie alle calunnie

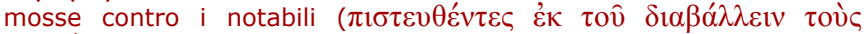
$\gamma v \omega \rho\{́(\mu) \varsigma)$. In effetti, alcune tirannidi si sono costituite in questo modo, quando ormai le città si erano accresciute (ท̌ $\delta \eta \tau \hat{\omega} v \pi$ ó $\lambda \varepsilon \omega v$

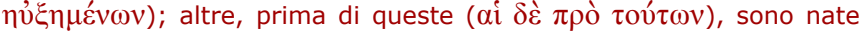
dai re che deviavano dai costumi tradizionali e aspiravano ad un

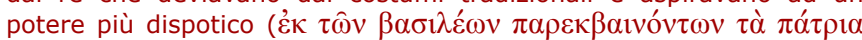

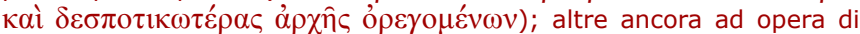
cittadini eletti alle cariche supreme: anticamente, infatti, i popoli isti-

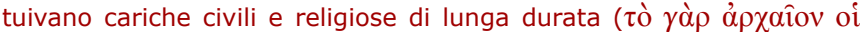

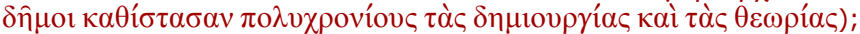
altre tirannidi, infine, emersero dalle oligarchie che eleggevano un solo individuo dotato di poteri sovrani sulle cariche più importanti ( $\alpha i \delta^{\prime}$

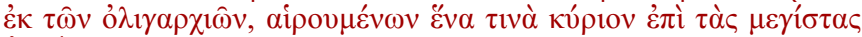
$\alpha \rho \chi \alpha ́()$ ). In effetti, attraverso questi modi, era possibile a tutti raggiungere facilmente I'obiettivo [...] dal momento che gli uni detenevano già la forza del potere regale, gli altri quella della carica pubblica ( $\delta i \alpha$

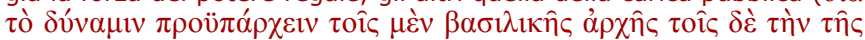
$\tau \imath \mu \hat{\eta} \varsigma) \gg$.

[20] In questo senso, III 18, 1288a 32-b 2; IV 2, 1289a 30-35; cfr. anche IV 7, 1293b 1-7 e, supra, n. 6. Al riguardo: ACCATTINO 1986, p. 59 s., 74 s.; Carsana 1990, p. 13; AccatTino 2000; Zizza 2012a, p. $170-171$.

[21] Cfr. III 14, 1285b 4-16 con il commento di Accattino \& CuRnis 2013, p. 220-221.

[22] III 14, 1285b 13.

[23] III 14, 1285b 5-8 (per la traduzione: AcCATtIno \& CURNIS 2013, p. 123). Sui re-patriarchi delle prime forme di comunità cfr. I 2, 1253a 15-27 con KeYt 1999, 143; Besso \& CuRnis 2011, p. 210-211. 
discorso e a renderlo più articolato, sviluppando o precisando I'enunciato posto in apertura (1310b 34-36), dove si afferma che, in passato (evidentemente), acquisivano lo statuto e il titolo di basileus coloro che avevano reso benefici alle poleis o agli ethne di appartenenza o - come alternativa per nulla valorizzata dalla critica (cfr. anche infra) - coloro che erano in grado di rendere benefici proprio perché epieikeis/gnorimoi e, quindi, per le loro qualità: axia, arete, genos, dynamis [24]. Si passa, così, dal segmento di partenza agli exempla (singoli o in serie), attraverso l'inserimento di altri più brevi e specifici enunciati, funzionali a declinare l'euergesia: nella fattispecie (e in ordine inverso), fondare città o impossessarsi di un territorio, liberare il popolo dall'oppressore e impedire che i concittadini diventino schiavi di guerra. Tra le forme che poteva assumere l'euergetein degli epieikeis (o degli gnorimoi), quelle che erano più importanti per un greco (e per Aristotele, evidentemente) riguardavano senza dubbio la eleutheria; non a caso, infatti, è a questo 'bene' che fanno riferimento le prime due categorie di euergetesantes e, quindi, i rispettivi exempla: solo dopo I'inserimento di Codro e di Ciro, eletti a 'paladini' della libertà dei loro popoli, compaiono in posizione meno enfatica i re dei Lacedemoni, dei Macedoni e dei Molossi, menzionati cumulativamente come esempi di benefattori di tutt'altro genere rispetto ai primi due personaggi.

Giunti a questo punto del nostro ragionamento, prima di procedere oltre, non sarà superfluo - per motivi di chiarezza - riproporre il testo che stiamo analizzando, mettendo in evidenza la struttura logico-argomentativa seguita da Aristotele:

- 1310b 34-36: «tutti coloro che beneficarono $\left(E^{1}\right)$ o che erano in grado di benificare le città o i popoli $\left(E^{2}\right)$ ottenevano questo onore » [enunciato principale $(E)$ ]

- 1310b 36-37: «alcuni per aver impedito che diventassero schiavi di guerra, $̋ \sigma \pi \varepsilon \rho$ Codro » [enunciato secondario (e1) + exemplum (ex.1)]

- 1310b 37-38: «altri per averli liberati, ఝَб $\varepsilon \rho$ Ciro» [enunciato secondario (e2) + exemplum (ex.2)]

[24] Cfr. supra, n. 10, 12, 16. Sulle valenze socio-politiche assunte nella Politica dagli appellativi gnorimos, epieikes e beltistos si rinvia a GASTALDI 1978, p. 88-90.

[25] NEWMAN 1902 [1973], p. 419 (cfr. anche infra, n. 31).

[26] Riporto, qui di seguito, quanto ho scritto altrove a proposito del rapporto tra Aristotele e la historia e, in particolare, sulla natura delle notazioni storiche della Politica: «La historia della Politica, così come I'opera è stata confezionata - prescindendo dalla sua stratigrafia - , è una storia 'allusa', ridotta all'essenziale e presentata sotto forma di brevi notazioni talvolta davvero cursorie, quasi sempre prive di paternità e di coordinate temporali, tranne rare eccezioni [...]. Una storia 'compendiata' [...] per la $\varphi$ i
- 1310b 38-40: «altri ancora per aver fondato città o essersi impossessati di un territorio, œ̋ $\pi \varepsilon \rho$ i re dei Lacedemoni, dei Macedoni e dei Molossi » [enunciato secondario (e3) + gruppo di exempla (ex.3)].

A ciascuna delle tre tipologie di euergetesantes ( $g$ li enunciati secondari: e1, e2, e3) corrisponde un solo exemplum (ex.1, ex.2) o un gruppo di exempla (ex.3):

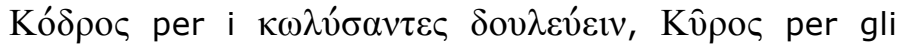

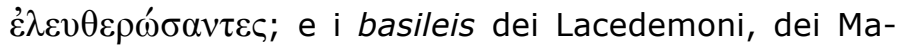

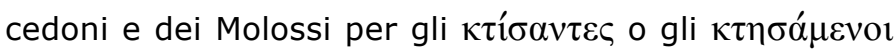
$\chi \omega ́ \rho \alpha \nu$. È, pertanto, ragionevole credere che ciascuna delle tre serie di exempla (le prime due formate, rispettivamente, da un solo caso; la terza - come si è detto - da tre) pertenga principalmente all'enunciato che la precede e che la introduce nel discorso. In questa prospettiva, anche l'impiego di œ̋ $\sigma \pi \varepsilon \rho+$ il nominativo (1310b 37, 38 e 39) potrebbe essere interpretato come un rivelatore della struttura del brano, dal momento che la formula ricorre tante volte quante sono le declinazioni

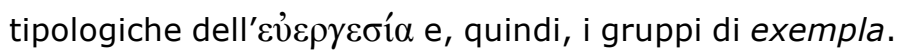
Nel caso specifico, dunque, l'enunciato di partenza (E) risulta direttamente correlato non tanto agli exempla (come, al contrario, ritengono Newman ed altri) [25], ma ai segmenti che precedono gli exempla medesimi (cioè - lo ribadiamo - gli enunciati secondari: e1, e2, e3) [26]. Pertanto, a restare escluso dal sistema di relazioni reciproche intercorrenti tra 'fatti' (gli exempla) e 'concetti' (gli enunciati secondari) è proprio il segmento di partenza (E) che, a tutto il discorso che segue, fa da premessa e da introduzione generale; qui, infatti, Aristotele non si limita a fare riferimento a coloro che 'diventarono' basileis per aver reso qualche beneficio $\left(E^{1}\right)$, ma menziona anche i benefattori 'in potenza' $\left(E^{2}\right)$ :

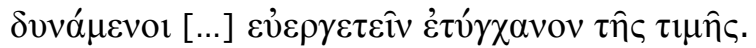

Gli exempla, dunque, ricoprono una specifica funzione esplicativa in riferimento alla categoria a cui sono abbinati, a prescindere dal fatto che si tratti di casi in cui I'acquisizione della time basilike sia avvenuta in seguito a un determinato beneficio reso al popolo (gli euergetesantes tout court; e tra questi, senz'altro, Ciro) o in virtù

quelle di natura etno-antropologica a quelle di tipo politico-militare) sono citate a sostegno della theoria, secondo un sistema di relazioni reciproche tra 'fatti' (cioè: exempla) e 'concetti' che in genere non segue una regola fissa o un modello univoco 'preconfezionato' (simmetria, prossimità, chiasmo, etc.), ma si rivela tutto sommato libero e, di volta in volta, imbastito in maniera tale che ad essere coinvolti nel medesimo sistema (fatti-concetti) si trovano ad essere non solo gli enunciati di partenza o i temi principali di una determinata sezione, ma anche le argomentazioni minori o secondarie generate dal ragionamento, attraverso un processo di gemmazione progressiva di concetti/idee e, quindi, - se ritenuto opportuno e necessario - dei relativi exempla» (ZIZZA 2012a, p. 135-136, per la citazione; e, per l'analisi di alcuni casi concreti, p. 162 s., 183 s.). Al riguardo, cfr. anche DE LUNA 2010, p. 61-63; DE LUNA 2014. 
della buona reputazione goduta e prima ancora di aver

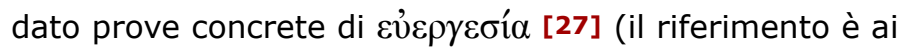
cosiddetti benefattori 'potenziali', categoria nella quale, a buon diritto, può rientrare Codro).

D'altra parte, se tutta la sezione della Politica di cui stiamo parlando è dedicata ai tropoi della euergesia e, quindi, alle praxeis degli euergetesantes, va da sé che anche nell'appendice esemplificativa del discorso l'attenzione di Aristotele si sia concentrata non tanto sulle figure dei protagonisti o sul titolo da questi posseduto nel momento in cui ciascuno di loro rese un beneficio alla propria polis o al proprio ethnos, quanto piuttosto sulle loro azioni: un tempo, ottimi candidati per la time basilike erano (o potevano essere) tutti gli epieikeis/gnorimoi che si comportavano - per esempio - come si comportò Codro quando era già re di Atene (cioè, oi $\mu \varepsilon \grave{\varepsilon} v \kappa \alpha \tau \grave{\alpha} \pi$ ó $\lambda \varepsilon \mu o v$

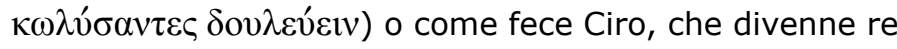
dei Persiani dopo che riuscì a liberare il suo popolo dalI'oppressione dei Medi (oi $\delta$ ' $\varepsilon \lambda \varepsilon v \theta \varepsilon \rho \omega ́ \sigma \alpha \nu \tau \varepsilon \varsigma$ ).

\section{L'EXEMPLUM DI CODRO}

Posta la questione in questi termini e tenuto conto del tipo di relazioni esistenti, nel caso specifico, tra I'enunciato principale e gli enunciati secondari (E-e1, E-e2, E-e3), da una parte, e tra questi ultimi che precedono gli exempla e gli exempla medesimi (e1-ex.1, e2-ex.2, e3-ex.3), dall'altra, proviamo ad analizzare il caso di Codro, che, tra tutti gli exempla del locus in esame, è quello che ha attirato maggiormente l'attenzione della critica, perché ritenuto poco pertinente al contesto.

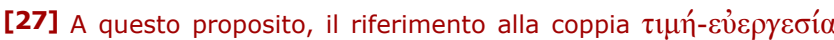
di Rhetorica I 5, 1361a 28-30 può fornire una autorevole e significativa conferma: «L'onore è il segno concreto di una reputazione d

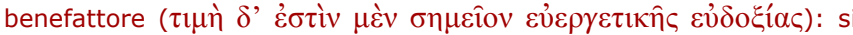
onorano giustamente e soprattutto quelli che già hanno fatto del bene

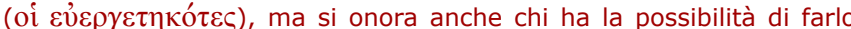

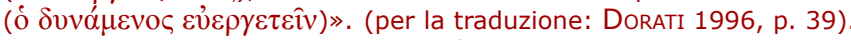
Cfr. anche Rhet. I 9, $1367 \mathrm{~b}$ 28-34. Più o meno sulla stessa linea Xen.,

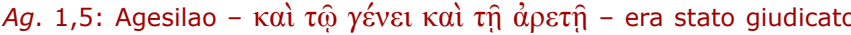
persona ineccepibile e degna della basileia ancor prima di assumere il governo della città.

[28] Cfr., rispettivamente, Plat., Symp. 208d; Lycurg., c. Leocr. 86. [29] Nello specifico, se parla di Dori in Hdt. V 76 e Strab. XIV 2,6 (ma cfr. IX 1,7, dove vengono menzionati anche gli Eraclidi); di Peloponnesii, invece, in Lycurg., c. Leocr. 84 (cfr. infra) e Paus. I 19,5; 39,4; VII 25,2. Come si dirà meglio in seguito (cfr. infra, nel testo e n. 41 s.), le prime due fonti ricordano I'invasione dell'Attica al tempo di Codro, ma non fanno alcun riferimento al sacrificio del re ateniese; Licurgo e Pausania, invece, collegano il ritiro degli invasori da Atene e dall'Attica allo stratagemma escogitato da Codro per farsi uccidere dai nemici e per salvare, quindi, la propria patria da una schiavitù certa. Tuttavia, se il Periegeta si limita a fare solo delle allusioni alla storia del sacrificio, Licurgo, al contrario, nella Contro Leocrate (84-87), ci fornisce una versione completa e dettagliata

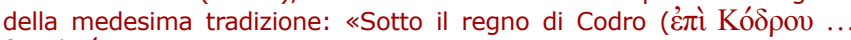
$\beta \alpha \sigma 1 \lambda \varepsilon v ́(v \tau 0 \varsigma)$, i Peloponesii, essendo stato il loro paese afflitto da carestia, deliberarono di muovere guerra alla nostra città e, dopo averne cacciato i nostri maggiori, dividersi il territorio. E anzi tutto mandarono a Delfi a interrogare il dio se avrebbero espugnato la città; e avendo il dio risposto loro che l'avrebbero espugnata se però non avessero ucciso
Personalmente, nonostante le opinioni contrarie di molti studiosi, sono convinto che le indicazioni - sia pur vaghe fornite da Aristotele a proposito del tipo di beneficio reso da Codro ai suoi concittadini non possano che alludere alla medesima storia a cui fanno riferimento (più) esplicito le fonti coeve alla Politica: il mitico re di Atene rese un beneficio alla patria sacrificando la sua vita $\dot{v} \pi \dot{\varepsilon} \rho \tau \hat{\eta} \varsigma$

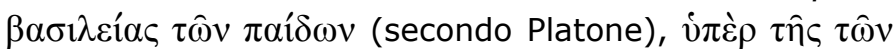

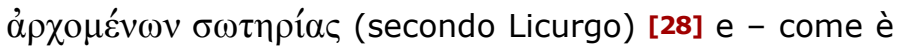
detto nel passaggio in esame - per impedire che il suo popolo fosse ridotto in schiavitù nel caso in cui avessero avuto la meglio i nemici (Dori/Peloponnesii) [29]. D'altra parte, se quanto detto sopra punta al vero, non mi sembra che sussistano ragioni sufficientemente valide per ammettere con Newman che esista una incongruenza tra l'exemplum relativo al sacrificio di Codro e il presunto enunciato di riferimento $(E)$, peraltro solo parzialmente preso in considerazione [30]: «According to the traditional account Codrus was already king of Athens when he delivered his country from a Dorian invasion by the sacrifice of his life, whereas Aristotle evidently takes him, if the text is correct, to have won a kingship by saving his country from enslavement» [31].

Stesso discorso per le ipotesi avanzate anche recentemente da quanti, continuando ad assumere come presupposto indiscusso e indiscutibile l'interpretazione fornita da Newman, hanno tentato di risolvere la (presunta) aporia o attraverso la valorizzazione di una storia in cui la morte di Codro era presentata in maniera completamente diversa rispetto alla versione 'canonica' relativa al sacrificio [32] o attraverso il 'recupero' di un idionimo diverso da quello tràdito nel testo della Politica e, nella fattispecie, quello

il re degli Ateniesi, Codro, mossero contro Atene. Cleomanti, uno di Delfi, venuto a conoscenza del responso, segretamente ne informò gli Ateniesi [...]. Avendo dunque i Peloponnesii invaso I'Attica, che fanno i maggiori vostri, o signori giudici? Non se n'andarono abbandonando il paese, come Leocrate, non lasciarono in balia dei nemici la terra che li nutrì e i templi, ma in numero esiguo, rinserrati, subivano l'assedio e resistevano per il bene della patria [...]. Narrano dunque che Codro, dopo aver avvertito gli Ateniesi che stessero ben attenti al momento in cui egli sarebbe caduto, indossata una veste da mendico per ingannare i nemici, uscito di soppiatto dalle porte, si desse a raccoglier sarmenti davanti alla città; ed essendosi a lui avvicinati due uomini usciti dall'accampamento nemico e chiedendo notizie sulle condizioni della città, egli ne abbattè uno con la falce avventandosigli contro; e il superstite, acceso d'ira contro Crodo e credendolo un mendico, tratta la spada I'uccise. Allora gli Ateniesi mandarono un araldo a chiedere il re per seppellirlo, dicendo loro tutta la verità: i Peloponnesii lo restituirono, ma comprendendo che non sarebbe stato loro più possibile occupare il paese, si ritirarono» (per la traduzione: MaLCOVATI 1977, p. 871-873).

[30] In genere, il concetto che viene poco valorizzato o del tutto trascurato è quello relativo alla cosiddetta euergesia in 'potenza' attribuita (e attribuibile) ad alcuni individui ritenuti virtuosi e superiori per axia, genos, dynamis, etc. (cfr. supra).

[31] NEWMAN 1902 [1973], p. 419. In questo senso anche SCHüTRUMPF \& GEHRKE 1996, p. 550.

[32] Cfr., per esempio, AubOnNet 1989, p. 204-205; HeSK 2000, p. 92, n. 31. La possibilità è ammessa anche da NEWMAN 1902 [1973], p. 420 come una alternativa all'ipotesi dell'errore (cfr. supra, n. 31). 


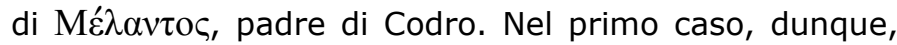
saremmo noi a non conoscere la tradizione che aveva in mente Aristotele; nel secondo, sarebbe il filosofo ad aver sbagliato: questi, infatti, avrebbe voluto alludere all'acquisizione del titolo di basileus da parte di Melantos - avvenuta in seguito ad una vittoria riportata sul re dei Beoti, che avevano invaso I'Attica [33] -, ma, per un lapsus, scrive il nome del figlio perché «more famous and even proverbial» [34].

A mio avviso, invece, entrambi i 'percorsi' indicati dalla critica sono da evitare: il testo in questione, infatti, non ha bisogno di essere emendato; né occorre tirare in ballo una «alternative version of his death [sc. Codrus] during the Dorians invasion» o pensare ad un «completely different exploit» [35]. Come si è detto, valorizzando, da una parte, la relazione biunivoca tra l'exemplum e il breve enunciato che lo precede (e1-ex.1) e, dall'altra, il riferimento ai benefattori 'in potenza' $\left(E^{2}\right)$ contenuto nell'enunciato principale (E), nulla osta a interpretare il riferimento a Codro come un'allusione al sacrificio del basileus [36]: una storia, questa, che per il pubblico della Politica doveva essere ormai piuttosto nota, se Aristotele può addirittura permettersi di evocarla tramite un cenno tanto cursorio quanto vago.

Nessuna incongruenza, dunque, tra exemplum e contesto. Ovviamente, non ho alcuna difficoltà ad ammettere la possibilità che in qualche passaggio della Politica il filosofo abbia potuto commettere errori, addurre esempi poco (o per nulla) pertinenti e fare riferimenti a tradizioni a noi (più o meno) sconosciute; nel caso specifico, tuttavia, sono convinto che l'errore è da imputare agli interpreti, non alla fonte. Per Aristotele, Codro è, innanzitutto, un modello di comportamento, perché scelse di agire per il bene del suo popolo. Se, nel momento in cui compì il sacrificio, era già re poco importa: il titolo, infatti, lo aveva acquisito perché - in quanto aristos e dotato (quindi) di axia, arete, genos e dynamis - era

[33] La notizia è tràdita, per esempio, da Strab. IX 1,7.

[34] Cfr. JACOBY 1954, p. 50 (per la citazione), 53-54. In questo senso, e tra le altre ipotesi, già NEWMAN 1902 [1973], p. 420; più recentemente, Carlier 1984, p. 360-361, n. 222; Steinbock 2011, p. 286, n. 36.

[35] HESK 2000, p. 92, n. 31.

[36] Il discorso varrebbe lo stesso anche se non si fosse eventualmente disposti a considerare il sistema di relazioni tra 'fatti' (exempla) e concetti (enunciati secondari) fin qui proposto; in questo caso, infatti, l'allusione al sacrificio di Codro sarebbe, nondimeno, da giudicare calzante proprio perché nell'enunciato principale si fa riferimento ai benefattori 'in potenza': e questo è un dato indiscutibile, che già di per sé basterebbe a risolvere la faccenda. Evidentemente, il problema della congruenza o meno dell'exemplum interessa solo il caso di Codro; negli altri exempla la questione non si pone e - aggiungerei - non si porrebbe lo stesso anche se si volesse continuare ad adottare la prospettiva indicata da Newman e seguita dai più: a seconda dei casi, infatti, risulta irrilevante o irrilevabile stabilire con quale segmento concettuale ciascun exemplum 'dialoghi' e faccia sistema. Il caso di Ciro, per esempio, si rivela pertinente sia all'enunciato di partenza (e, in particolare, al primo concetto che compare nel segmento: ö $\pi \alpha \nu \tau \varepsilon \varsigma .$. stato ritenuto in grado di poter rendere un beneficio alla patria qualora si fosse presentata l'occasione. E I'occasione non mancò. Codro si lascia uccidere dai nemici per ottenere, in cambio della vita, la libertà dei suoi sudditi e si rivela, quindi, un benefattore a tutti gli effetti: con la sua 'immolazione', riesce a dimostrare - semmai ce ne fosse stato bisogno - di non aver disatteso aspettative e fiducia di quanti lo riconobbero come proprio monarchos ancor prima che avesse compiuto il sacrificio della propria vita, l'impresa più degna di essere annoverata tra le cosiddette praxeis euergetesantes.

\section{IL SACRIFICIO DI CODRO: LE FONTI}

Che la storia del sacrificio di Codro sia stata narrata in maniera distesa per la prima volta da Ellanico [37] è probabile (e forse anche possibile), ma non certo: risulta difficile, infatti, stabilire con sicurezza se siano da attribuire effettivamente all'attidografo tutte le informazioni riportate dalla fonte tralatrice (nella fattispecie, lo scoliasta a Plat., Symp. 208d) o solo quelle che compaiono all'inizio del lungo frammento e che riguardano la genealogia di Codro [38]. Nessun dubbio, invece, sul fatto che, del sacrificio, la versione più completa sia - lo ribadiamo - quella riferita da Licurgo [39]. Infatti, dalla Contro Leocrate, oltre che dal frammento di Ellanico (o dallo scolio), apprendiamo dettagli altrimenti sconosciuti e, in qualche caso, solo parzialmente noti da altre fonti, come, per esempio, l'uso da parte di Erodoto del nome di Codro per identificare la prima spedizione che i Dori organizzarono contro I'Attica [40]; la notizia riferita da Ferecide sul travestimento adottato dal re per non farsi riconoscere dai nemici [41]; l'epiteto $\delta \rho v \eta \kappa o ́ \pi o c ~ u t i l i z z a t o$ da Licofrone al posto del nome proprio del basileus [42]; l'allusione di Pausania all'oracolo delfico in virtù del quale, morto Codro, i Peloponnesii persero ogni speranza di

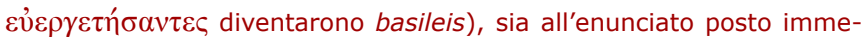
diatamente prima della citazione del nome (oi $\delta$ ' $\varepsilon \lambda \varepsilon v \theta \varepsilon \rho \omega ́ \sigma \alpha \nu \tau \varepsilon \varsigma$ ): Ciro divenne re avendo reso un beneficio ai Persiani (liberò, infatti, il suo popolo dai Medi). I basileis delle linee 1310 b 39-40, invece, proprio per il modo in cui sono menzionati, lasciano aperta ogni possibilità di lettura e si prestano ad essere interpretati in un senso o nell'altro, a seconda del tipo di relazioni reciproche che si è disposti ad ammettere come operante nell'intera sezione e, quindi, soprattutto tra l'exemplum di Codro - che è appunto quello più problematico - e gli enunciati che lo precedono: mi riferisco, ovviamente, sia all'enunciato principale $\left(E^{1}+E^{2}\right)$, sia all'enunciato secondario $(e 1)$.

[37] Hellan., FGrHist 4 F $125=323 a$ F 23.

[38] Sulla questione cfr. AmBAgLio 1980, p. 164-165.

[39] Cfr. supra, n. 29.

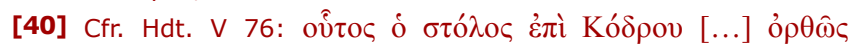

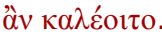

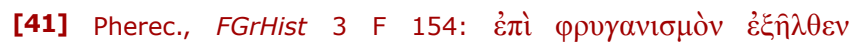

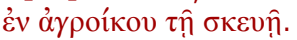

[42] Lycoph., Alex. 1378-1379 HuRST \& KOLDE 2008. 
vittoria e, quindi, si ritirarono dall'Attica [43]. Va da sé che, in assenza della testimonianza di Licurgo e di quella che si attribuisce a Ellanico, sarebbero risultate quasi del tutto oscure anche le allusioni che gli scrittori dal V secolo a.C. in poi hanno fatto a Codro e alla sua basileia soprattutto per poter trattare il tema del popolamento della Ionia e per menzionare, quindi, i Codridi in quanto ecisti e/o promotori del movimento migratorio iniziato

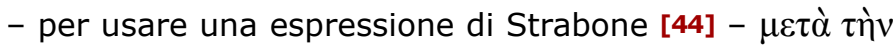

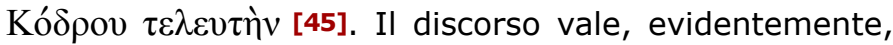
anche per quelle fonti letterarie che - come i due testi di riferimento - mostrano un interesse precipuo, non tanto (o non solo) per le ktiseis ioniche, quanto piuttosto per la figura eroica e paradigmatica del mitico re di Atene. È così, per esempio, per Platone [46], per Aristotele e, in genere, per gli autori coevi e successivi a questi: dal IV secolo a.C. in poi risulta sicuramente più facile trovare la menzione del basileus tra i benefattori della patria e, in quanto tale, tra gli exempla virtutis del passato [47]. La stessa cosa non può dirsi con la medesima sicurezza per il V secolo, anche se è alle fonti di questo periodo (ma non alle precedenti) che risalgono quelle che per noi costituiscono le attestazioni più antiche del nome di Codro. In questi casi - prescindendo, per le ragioni esposte sopra, dal frammento di Ellanico [48] -, il riferimento specifico a Codro sembrerebbe avere (ancora e prevalentemente) una funzione di 'servizio', visto che è soprattutto per introdurre altri personaggi che viene evocato il basileus: si pensi ai già menzionati Codridi-oikistai [49], ma anche ai Pisistratidi, che di Codro e di suo padre Melanto si consideravano discendenti [50].

Sulla base di quanto detto, si può ammettere (almeno come possibilità) che la mitica figura di Codro (al quale lo stesso Erodoto non sembra attribuire alcun gesto eroico) abbia subito nel corso degli anni un processo tale per cui solo da un certo momento in poi (dopo Erodoto?) si iniziò a parlare del personaggio anche a prescindere dalle imprese dei 'figli' (in senso lato) o indipendentemente dal fatto che qualcuno dei cosiddetti Codridi fosse ricordato. Il Codro di metà V e, sicuramente, di IV secolo (e oltre) avrebbe, dunque, acquisito il ruolo del protagonista

[43] Paus. VII 25,2.

[44] Strab. XIV 2,6.

[45] A proposito dei Codridi e del popolamento della Ionia cfr., per esempio, Hdt. I 147,1; IX 97,1; Pherec., FGrHist 3 F 155; Strab. XIV 1,3 s.; Paus. VII 2,1 s.; IX 37,8. Sull'argomento specifico si rinvia a Momigliano 1933 [1975], p. 373 s.; CASSOLA 1957, p. 31 s., 84 s., 126-127; Moggi 1996; SchütrumpF \& Gehrke 1996, p. 550; Moggi \& Osanna 2000, p. $187 \mathrm{~s}$.

[46] Plat. Symp. 208c-d.

[47] Cfr., tra gli altri, Conon, FGrHist 26 F 1 (26); Paus. VIII 52,1 (ma si confronti anche I 19,5 con Lycurg., c. Leocr. 84 e IG II/III ${ }^{2}$ 4258) e, ancora, Paus. X 10,1-2 (per la segnalazione di luoghi o di 'oggetti' legati al personaggio e al suo sacrificio). assoluto e, nello stesso tempo, lo statuto del sovranobenefattore, che prima non possedeva o, più prudentemente, che prima non mostrava. In altre parole, se è possibile che alcuni segmenti del racconto fossero noti fin dal V secolo a.C. (o già anche nel VI) [51], non si può dire altrettanto né a proposito della elezione del basileus a modello da imitare (in quanto benefattore e 'patriota'), né relativamente alla trama che doveva tenere insieme i singoli elementi del logos (era già quella tràdita da Ellanico e da Licurgo?). Certo è che almeno le linee essenziali della vicenda (spedizione dei Dori contro gli Ateniesi, fallimento dell'impresa per l'intervento di Codro etc.) dovevano essere note fin dai tempi di Erodoto e, con ogni probabilità, anche prima di Erodoto. Progressivamente, attraverso un percorso del quale non riusciamo a seguire tutte le tappe, la tradizione (forse con Ellanico; probabilmente anche con Ferecide) dovette giungere ad un livello tale di gestazione/sistemazione da dare vita a una parallela versione 'codrocentrica' della storia, che non tarderà ad occupare «a prominent position in Athenian mythology» [52]: in questa direzione puntano anche le poche testimonianze iconografiche in nostro possesso e, per esempio, la coppa del cosiddetto Pittore di Codro [53] e il donario degli Ateniesi a Delfi segnalato da Pausania [54]. Evidentemente, al tempo della pubblicazione del decreto del 418/7 a.C. relativo a Codro e al santuario dedicato a lui, a Neleo e a Basile ( $I G \mathrm{I}^{3}$ 84), il 'capitolo' della tradizione sul basileus poteva dirsi ormai completato, così come il ritratto del personaggio e il significato della sua azione: di qui in avanti, il sacrifico di Codro e il beneficio reso al popolo assumerà toni decisamente proverbiali [55]. Già a Platone e ad Aristotele, dunque, non occorreva insistere sulla vicenda; bastava semplicemente accennarla (come di fatto mostrano di fare) perché fossero chiari a tutti gli elementi omessi o allusi (antefatti, conseguenze e relazioni tra gli eventi): nella fattispecie, la connessione tra la morte di Codro e il fallimento dell'impresa dei nemici; il travestimento del re per non farsi riconoscere dagli avversari; la provocazione del nemico; I'uccisione del basileus; la scoperta della vera

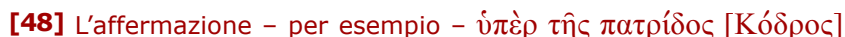

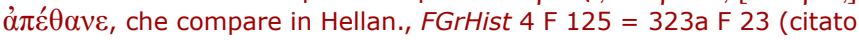
supra, n. 37), risale all'attidografo o alla fonte tralatrice?

[49] Si veda quanto detto supra nel testo e alla n. 45

[50] Cfr., per esempio, Hdt. V 65,19.

[51] In questo senso sembrerebbe puntare il legame parentelare stabilito dai Pisistratidi con la coppia Melanto-Codro (cfr. supra, n. 50).

[52] Steinbock 2011, p. 286.

[53] Cfr. BeAzley 1963, p. 1268, n. 1.

[54] Cfr. Paus. X 10,1-2. Sul donario e sul passo della Periegesi cfr., tra gli altri, Vidal-Naquet 1963; Jacquemin 1999, p. 186 s.; Neer 2004, p. 80 s.

[55] In questo senso anche SteinBock 2011, p. 286, n. 34 
identità dell'individuo che si era lasciato ammazzare; il ritiro dei nemici e il pericolo scampato.

Coerentemente, il medesimo stile 'allusivo' avrebbe potuto essere adottato anche dal contemporaneo dei due filosofi, Licurgo. È molto probabile, tuttavia, che l'oratore abbia deciso di narrare l'aneddoto in maniera distesa non per far conoscere una storia che ancora non si conosceva (o che si conosceva in modo lacunoso), ma soprattut-

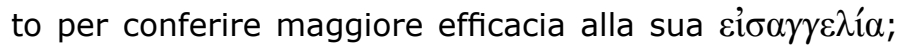
tanto più in dettaglio veniva riferito il logos, tanto più convincenti e d'effetto potevano suonare le sue parole e, quindi, le accuse rivolte a Leocrate, che nulla aveva in comune con l'amore per la patria posseduto da Codro, $\tau \hat{\omega} v \kappa \alpha \lambda \hat{\omega} \nu$ ع́ $\rho \gamma \omega \nu \pi \alpha \rho \alpha ́ \delta \varepsilon \imath \mu \alpha$ : «Ora, questa sarebbe davvero la cosa più strana, che, mentre i vostri maggiori affrontarono la morte perché la patria non fosse inglorio-

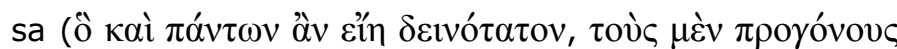

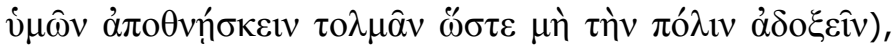
voi non abbiate a punire quelli che la disonorano ( $\dot{v} \mu \hat{\alpha} \varsigma$

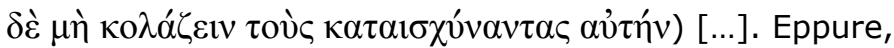
o signori, a voi soli tra gli Elleni non è lecito trascurare

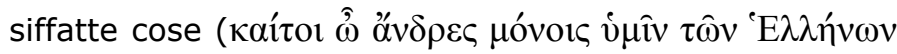

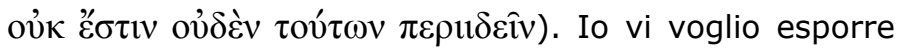

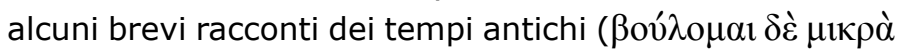
$\tau \hat{\omega} v \pi \alpha \lambda \alpha \iota \hat{\omega} v$ vं $\mu \hat{\imath} v \delta 1 \varepsilon \lambda \theta \varepsilon \hat{\imath} v)$, ai quali voi come a esempi

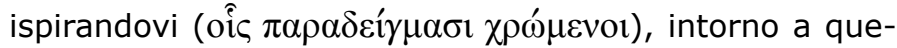
sto caso e agli altri tutti meglio delibererete ( $\alpha \alpha \grave{i} \pi \varepsilon \rho \grave{i}$

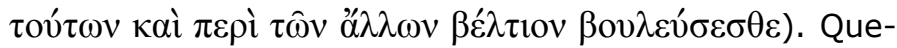
sto grandissimo vantaggio ha la città vostra, che di belle

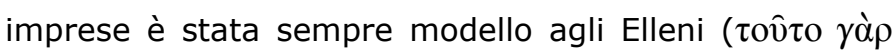

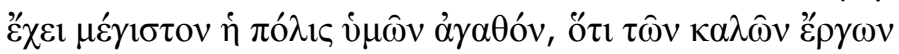

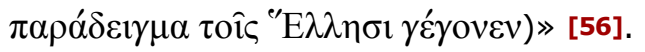

[56] Lycurg., c. Leocr. 82-83 (cfr. anche supra, n. 29). In generale, sul mito di Codro, sui personaggi a lui collegati e su altre fonti cfr. - oltre alla bibliografia citata supra e nel recentissimo lavoro di SteinBock 2011 - CARLIER 1984, p. 359 s., 432 s.; RoBertSON 1988, p. 201 s., 224 s.; Aubonnet 1989, p. 204-205; SimON 1992.

Accattino, P., 1986, L'anatomia della città nella Politica di Aristotele, Torino.

Accattino, P., 2000, «Il regime degli uomini perfettamente virtuosi: aristocrazia e costituzione ottima nella Politica di Aristotele», Etica \& Politica 2 (http://www2.units.it/etica/).

Accattino, P. \& Curnis, M., 2013, Aristotele, La Politica, Libro III, Roma (Istituto italiano per la storia antica: Aristotele, La Politica, 2, «L'Erma» di Bretschneider, dir. L. Bertelli \& M. Moggi).

Ambaglio, D., 1980, L'opera storiografica di Ellanico di Lesbo, Pisa (Ricerche di Storiografia Antica, II), p. 1-192.

Aubonnet, J., 1989, Aristote, Politique, Livres V et VI, $2^{\mathrm{e}}$ éd. (1 $1^{\text {re }}$ éd. 1973), Paris (CUF).

Besso, G. \& Curnis, M., 2011, Aristotele, La Politica, Libro I, Roma (Istituto italiano per la storia antica: Aristotele, La Politica, 1, «L'Erma» di Bretschneider; dir. L. Bertelli \& M. Moggi).

CARLIer, P., 1984, La royauté en Grèce avant Alexandre, Strasbourg.

Carsana, C., 1990, La teoria della 'costituzione mista' nell'età imperiale romana, Como.

CAssola, F., 1957, La Ionia nel mondo miceneo, Napoli.

De LunA, M. E., 2010, «Le metabolai nei regimi oligarchici: struttura argomentativa e uso dei dati storici nell'exemplum di Eritre (Pol. V 6 1305a37-1305b23)», in M. Polito \& C. Talamo (ed.), La Politica di Aristotele e la storiografia locale, Tivoli, p. 47-63.

De LunA, M. E., 2014, «Demagoghi e mutamenti costituzionali: alcune riflessioni (Aristot., Pol. 1304b19-1305a36)», Incidenza dell'Antico 11, c.d.s.

DoratI, M., 1996, Aristotele, Retorica, Milano.

García Gual, C. \& Pérez Jiménez, A., 1986 [2003], Aristóteles, Política, 9a reimpresión (1a imp. 1986), Madrid.

GASTALDI, S., 1978, «Lo spoudaios aristotelico tra etica e poetica», Elenchos 8, p. 63-104.

Hesk, J. P., 2000, Deception and Democracy in Classical Athens, Cambridge.

HuRst, A. \& Kolde, A., 2008, Lycophron, Alexandra, Paris.

JAсовY, F., 1954, Die Fragmente der Griechischen Historiker (FGrHist), Supplement III b: A Commentary on the Ancient Historians of Athens (Nos. 323a-334), II, Leiden.

JacQuemin, A., 1999, Offrandes monumentales à Delphes, Athènes (Bibliothèque des Écoles Françaises d'Athènes et de Rome 304). 
KeYT, D., 1999, Aristotle, Politics, Books V and VI, Oxford.

Laurenti, R., 1973 [2005], Aristotele, Politica, 7a rist., (1a imp. 1973), Roma - Bari.

López Barja de Quiroga, P. \& García Fernández, E., 2005, Aristóteles, Política, Madrid.

Malcovati, E., 1977, Licurgo, Contro Leocrate, in M. Marzi \& P. Leone \& E. Malcovati (ed.), Oratori Attici Minori, I: Iperide, Eschine, Licurgo, Torino.

MoggI, M., 1996, «L'excursus di Pausania sulla Ionia», in J. Bingen (éd.), Pausanias Historien, Vandœuvres - Genève (Entretiens Hardt 41), p. 79-105.

Moggi, M. \& OsannA, M., 2000, Pausania, Guida della Grecia VII. L'Acaia, Milano (Fondazione Lorenzo Valla / Arnoldo Mondadori).

Momigliano, A., 1933 [1975], «Questioni di storia ionica arcaica», SIFC 10, p. 259-297 (rist.: 1975), [Quinto contributo alla storia degli studi classici e del mondo antico, Roma, p. 369-402].

Neer, R., 2004, «The Athenian Treasury at Delphi and the Material of Politics», ClAnt 23, p. 63-93.

Newman, W. L., 1902 [1973], The Politics of Aristotle, IV, Oxford (reprint: 1973, New York).

Robertson, N., 1988, «Melanthus, Codrus, Neleus, Caucon: Ritual Myth as Athenian History», GRBS 29, p. $201-261$.

Ross, W. D., 1957, Aristotelis Politica, Oxford.

Schütrumpf, E. \& Gehrke, H.-J., 1996, Aristoteles, Politik, Buch IV-VI, III, Berlin.

Simon, E., 1992, s. v. Kodros, Lexicon Iconographicum Mythologiae Classicae VI.1, p. 86-88.

Steinbock, B., 2011, «A Lesson in Patriotism: Lycurgus' Against Leocrates, the Ideology of the Ephebeia, and Athenian Social Memory», ClAnt 30, p. 279-317.

Viano, C. A., 1952 [2002], Aristotele, Politica, Torino, 1952 (rist.: Milano, 2002).

Vidal-Naquet, P., 1963, «Une énigme à Delphes. A propos de la base de Marathon (Pausanias, X, 10, 1-2)», RH 238, p. $281-302$.

ZızzA, C., 2012a, «Dalla cacciata di Trasibulo all'avvento di Dionisio il Vecchio: la parentesi 'repubblicana' di Siracusa nella Politica di Aristotele», in M. Polito \& C. Talamo (ed.), Istituzioni e costituzioni in Aristotele: tra storiografia e pensiero politico, Tivoli, p. 131-188.

ZızzA, C., 2012b, «Tiranni greci e despoti orientali nella Politica di Aristotele: Periandro e Sardanapalo», Incidenza dell'Antico 10, p. $177-193$. 